\title{
Combining Passive Thermography and Acoustic Emission for Large Area Fatigue Damage Growth Assessment of a Composite Structure
}

\author{
Joseph N. Zalameda*a, Michael R. Horne ${ }^{\mathrm{b}}$, Eric I. Madaras ${ }^{\mathrm{a}}$, and Eric R. Burke ${ }^{\mathrm{a}}$, \\ ${ }^{a}$ NASA Langley Research Center Hampton, VA 23681-2199; \\ ${ }^{b}$ National Institute of Aerospace NASA Langley Research Center Hampton, VA 23681-2199
}

\begin{abstract}
Passive thermography and acoustic emission data were obtained for improved real time damage detection during fatigue loading. A strong positive correlation was demonstrated between acoustic energy event location and thermal heating, especially if the structure under load was nearing ultimate failure. An image processing routine was developed to map the acoustic emission data onto the thermal imagery. This required removing optical barrel distortion and angular rotation from the thermal data. The acoustic emission data were then mapped onto thermal data, revealing the cluster of acoustic emission event locations around the thermal signatures of interest. By combining both techniques, progression of damage growth is confirmed and areas of failure are identified. This technology provides improved real time inspections of advanced composite structures during fatigue testing.
\end{abstract}

Keywords: Thermal nondestructive evaluation, fatigue damage detection, aerospace composite inspection, acoustic emission, passive thermography

\section{INTRODUCTION}

During composites load testing, the measurement of the onset and growth of damage is necessary to test structural designs and to validate damage prediction models [1,2]. Understanding how a structure fails, will enable optimal designs for improved safety and performance of the part during its life cycle. Testing of these parts utilizes a building block approach where composite coupons and sub-components are tested [3]. The goal is to eventually test full scale composite fuselage and wing structures and therefore fully validate progressive damage analysis models. For a given composite design, understanding how damage initiates and grows improves confidence in predicting fatigue life. Inspection methods are required to measure damage initiation and growth in advanced composite structures during controlled load testing. The significant damage modes for composite structures are delamination/debonding, fiber breaks, and through-the-thickness matrix cracking.

In-situ inspection methods are required, since typically the composite part is wired with strain gauges and fiber optic sensors. In-situ inspection methods are more time efficient since the part does not need to be physically removed for inspection. Real time in-situ nondestructive evaluation (NDE) techniques are used to track the damage. When damage growth is detected, the loading was stopped for a more detailed inspection using non-immersion ultrasound. The nonimmersion ultrasound is used to provide a detailed assessment of the damage growth through-the-thickness and thus ultimately provided a better understanding of damage progression modes that can lead to ultimate failure [4]. Some methods for real time in-situ inspection during loading have involved acoustic emission, passive thermography, digital image correlation, and fiber optic. Each of these techniques has some limitations.

For example, acoustic emission can detect the onset of damage and location, but cannot determine the exact size of the defect or damage depth. Digital image correlation measures the out of plane displacement changes due to damage under loading, however, the damage shape and depth cannot be measured. Fiber optics can measure changes in strain in real time, but again cannot measure the shape or depth of the damage. Passive thermography is a real time inspection technique that can detect defect location and shape; however, smaller defects (fiber breaks and matrix cracking) that are deeply buried can be difficult to detect. By combining thermography with acoustic emission data, damage growth can be more accurately detected and therefore provides a more detailed real time inspection. In the past there have been

*joseph.n.zalameda@nasa.gov; phone 1 757-864-4793; fax 1 757-864-4914; http://nde.larc.nasa.gov 
numerous studies combining thermography with acoustic emission [5-8]. Our technique is unique wherein the acoustic emission data using an array of acoustic emission sensors are mapped onto the thermal imagery. To map the acoustic emission data, image processing is required on the thermal imagery. The image processing requires contrast enhancement, image rotation, and removal of optical lens distortions. Mapping the acoustic emission events onto the thermal images provides confirmation of damage growth at areas of heating and where ultimate failure will occur. This inspection technique will provide a measurement capability to detect damage (location and size) for improved structures testing. Multiple infrared cameras and multiple acoustic emission sensors can be employed for large scale testing of structures. With this strategy, damage progression leading to failure can be more accurately determined and help to validate damage prediction models.

\section{SAMPLE DESCRIPTION AND MEASUREMENT SYSTEMS}

\subsection{Composite Structure Tested}

The hat stiffened composite panel flat side, stringer side before instrumentation, stringer side after instrumentation, and acoustic emission sensor locations (stringer side) are shown in Figures 1a, 1b, 1c, and 1d respectively. The panel skin is 17 plies with a thickness of $0.32 \mathrm{~cm}$. The hat flange is 11 plies with a thickness of $0.21 \mathrm{~cm}$. Barely visible impact damage (BVID) were placed at 4 locations (I1, M1, I2, M2) on the panel skin between the hat stiffeners. The BVID was used to simulate existing delamination damage and serves as damage growth sites. Cyclic compressive loads were applied from $-20,000$ pounds up to $-150,000$ pounds at $2 \mathrm{~Hz}$ using the setup shown in Figure 2. High speed digital image correlation cameras were used and required illumination using red light emitting diode (LED) lights. The LED lights were not a problem for the infrared cameras which operated in the mid infrared waveband. The load was applied from the bottom while the top was held stationary. Passive thermography was used to track the damage during loading on both the flat and stringer sides. Shown in Figure 1d are the locations of the acoustic emission sensors in relation to the BVID. The acoustic emission sensors were used for real time detection of acoustic events indicating damage growth.

(a) Flat Side

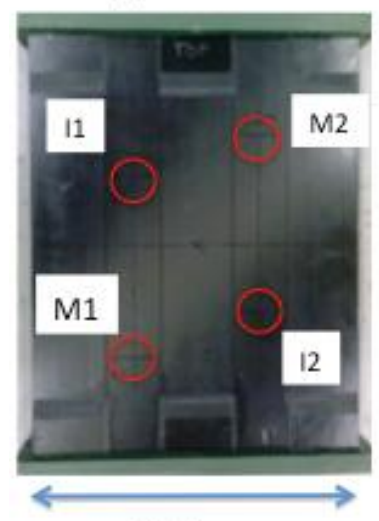

$43.8 \mathrm{~cm}$ (b) Stringer Side

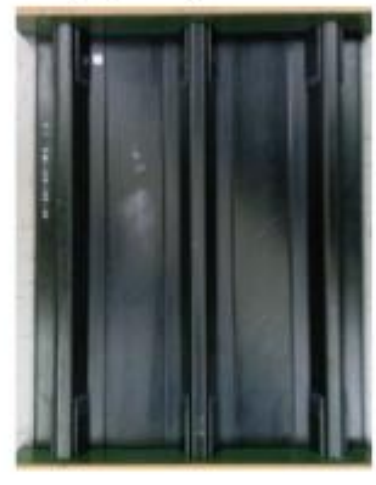

(c) Instrumented Panel

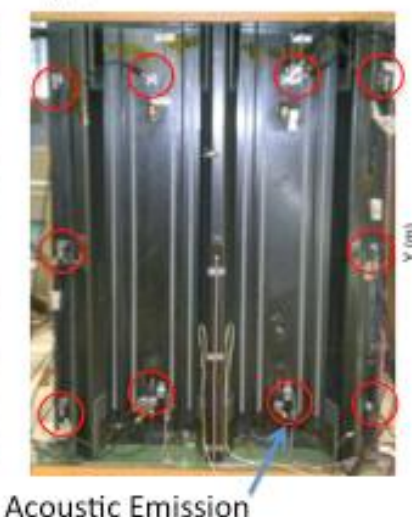

Acoustic Emission

Sensors (d) AE Sensor Location Stringer Side

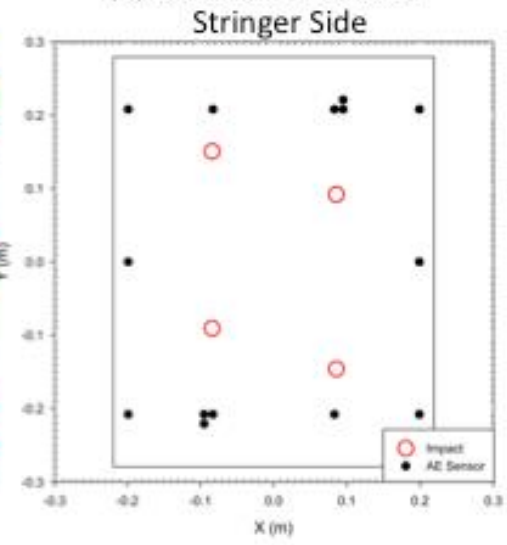

Figure 1. Three-stringer hat stiffened panel used for fatigue load testing.

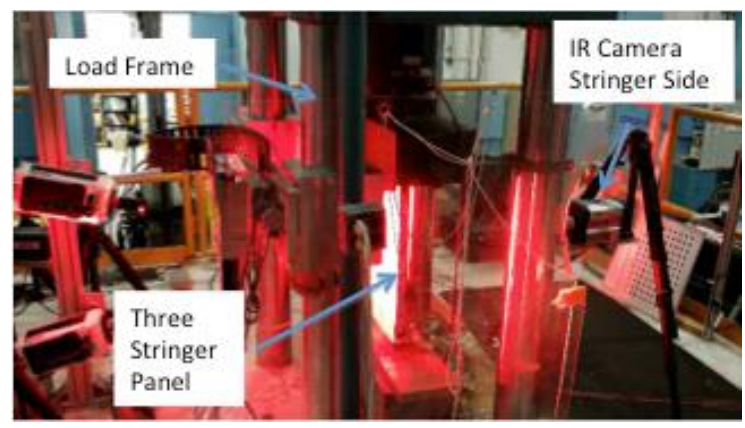

Figure 2. Fatigue loading test setup for three-stringer panel. 


\subsection{Acoustic Emission Sensors}

Fourteen acoustic emission sensors were bonded to the composite panel on the stringer side. They were connected to a data acquisition computer via Digital Wave TM PA0 preamp/line drivers and a Digital Wave TM FM1 signal conditioning 16 channel amplifier for capture of acoustic data. The acoustic emission computer also captured the load for synchronization. Acoustic emission systems collect structure-borne sound, typically not audible, in the ultrasonic frequency band (approx. 50 to $500 \mathrm{kHz}$ ), generated by dynamic displacements such as damage initiation and growth at picometer scales [9]. Pre-cursors to failure are monitored in real-time. The total signal energy, SE, of an acoustic signal is given as:

$$
S E=\sum_{i=1}^{n} V_{i}^{2} t
$$

where $V_{i}$ is the signal voltage, $i$ is the time reference point, $n$ is the number of time points in the signal, $\Delta \mathrm{t}$ is the sampling time per point. Trends of the energy over time, or load, are used to identify and track damage development. Signals from the distributed array of sensors are acquired simultaneously when any one sensor threshold triggers. This allows calculating the source or event location $[10,11]$. This is shown in Figures $3 \mathrm{a}$ and $3 \mathrm{~b}$ where the acoustic emission events are shown for different fatigue tests. The accumulated acoustic events for Fig. 3a were for approximately 50 seconds and for Figure $3 \mathrm{~b}$ was for 16.5 seconds. As the structure nears failure the accumulated acoustic events increases as a function of time as shown in Figure $3 \mathrm{~b}$.

(a) Flat Side of Panel $97.3 \%$ of Life

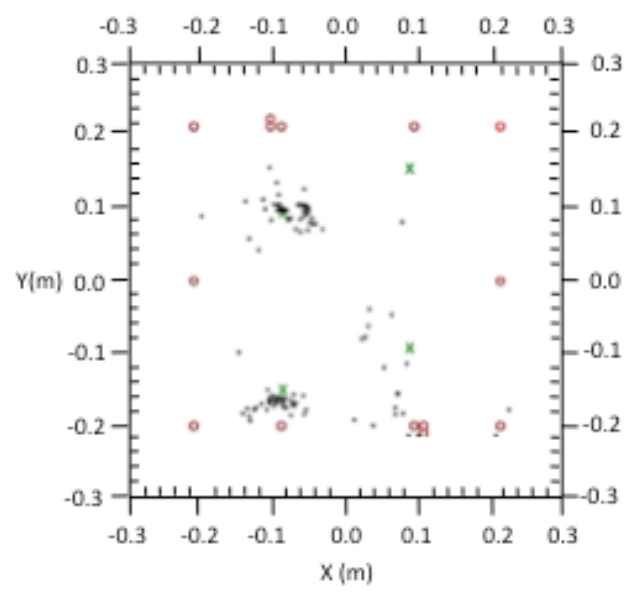

(b) Stringer Side of Panel $99.9 \%$ of Life

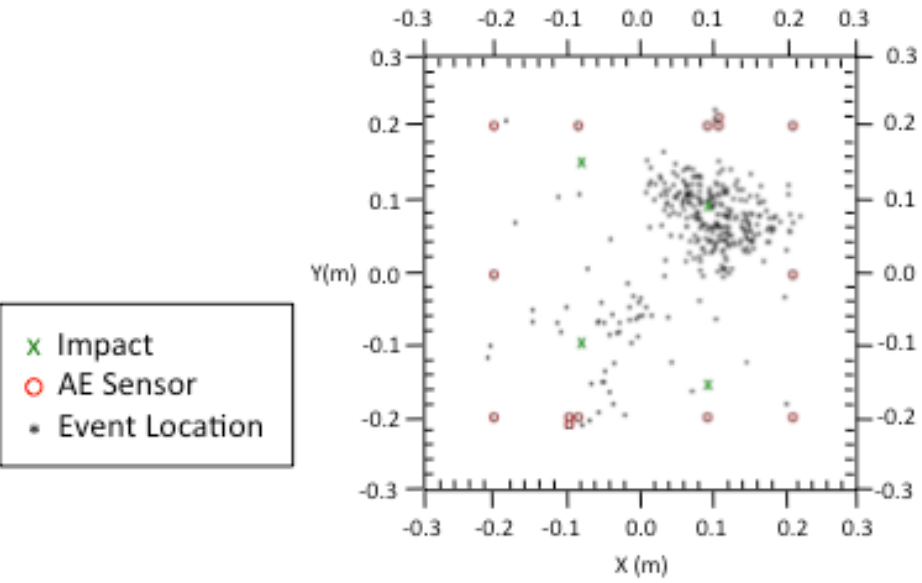

Figure 3. Three-stringer panel acoustic emission event locations.

\subsection{Passive Thermography}

The thermal measurement setup is shown in Figure 4. The basic system consists of two IR cameras operating in the 3-5

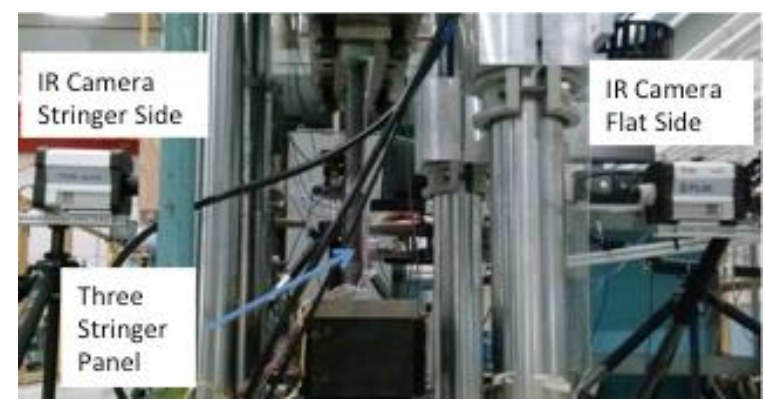

Figure 4. Three-stringer panel setup for passive thermography inspection of both flat and stringer sides. 
micrometer IR band and an image data acquisition computer. The IR cameras were both configured with $25 \mathrm{~mm}$ germanium optics. The focal plane array sizes for the cameras were from 640x512 to 1024x1204. The passive inspection captured the thermal variations during the fatigue loading. The setup required a Plexiglas ${ }^{\circledR}$ shield to contain fragments if ultimate failure occurred. The IR cameras were located behind the shield with the lens positioned in front of a viewing hole. The added benefit of the shield is it filters out spurious IR background sources. The cameras were synchronized using the load signal from the test machine. The sinusoidal load signal was offset corrected and the zero crossover points were used to produce a TTL trigger signal. The trigger signal was converted to a series of pulses using a signal generator. The IR cameras were operated at a frequency range of 16 to $90 \mathrm{~Hz}$ depending on acquisition time duration. Higher frequencies were used to capture the damage progression as the panel approached ultimate failure. Example unprocessed thermal images of the flat side are shown in Figure 5. The bright areas indicate the heated damaged sites.
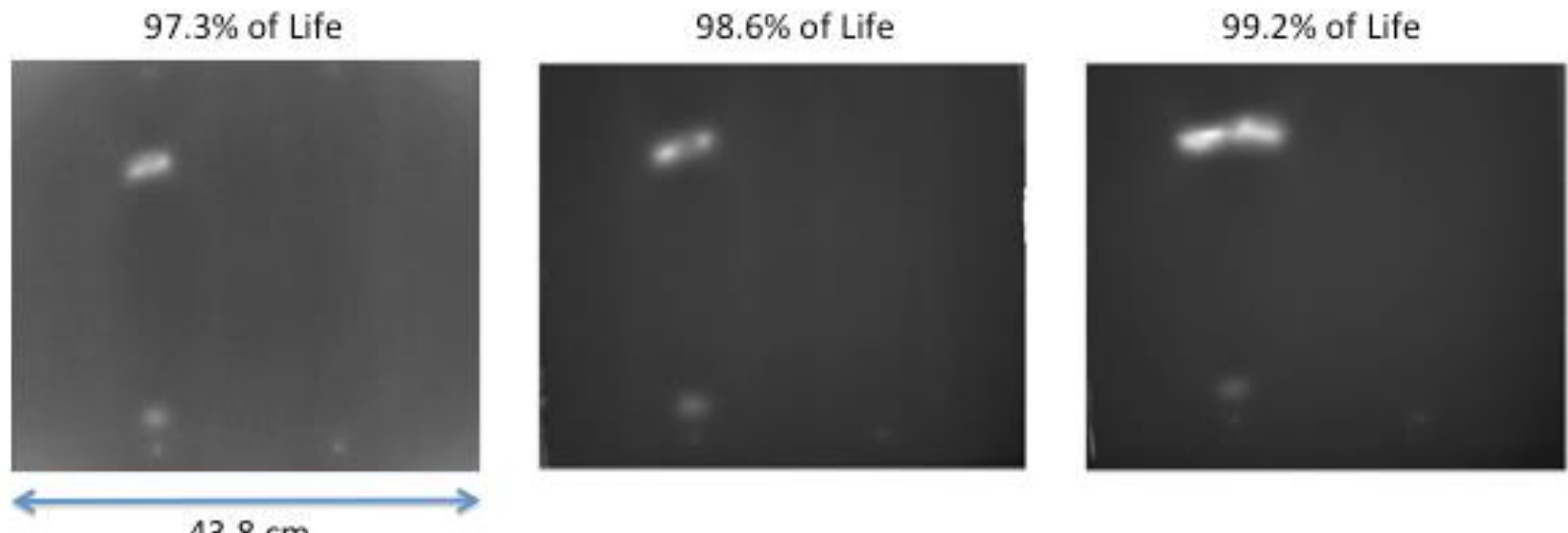

$43.8 \mathrm{~cm}$

Figure 5. Unprocessed thermography images (flat side) of damage growth as a function of remaining fatigue life.

\section{MAPPING ACOUSTIC EMISSION EVENTS WITH THERMOGRAPHY IMAGERY}

\subsection{Synchronization to Loading}

Synchronizing the timing of the acoustic emission and thermography acquisition to the applied load is necessary to match the acoustic events with the thermal data frame by frame. Since the damage formation will occur during the application of compressional forces, the acquired acoustic emission and thermal indications are confirmed and helps to eliminate false signals. This is shown in the left image of Figure 6 where the total acoustic energy, measured from all
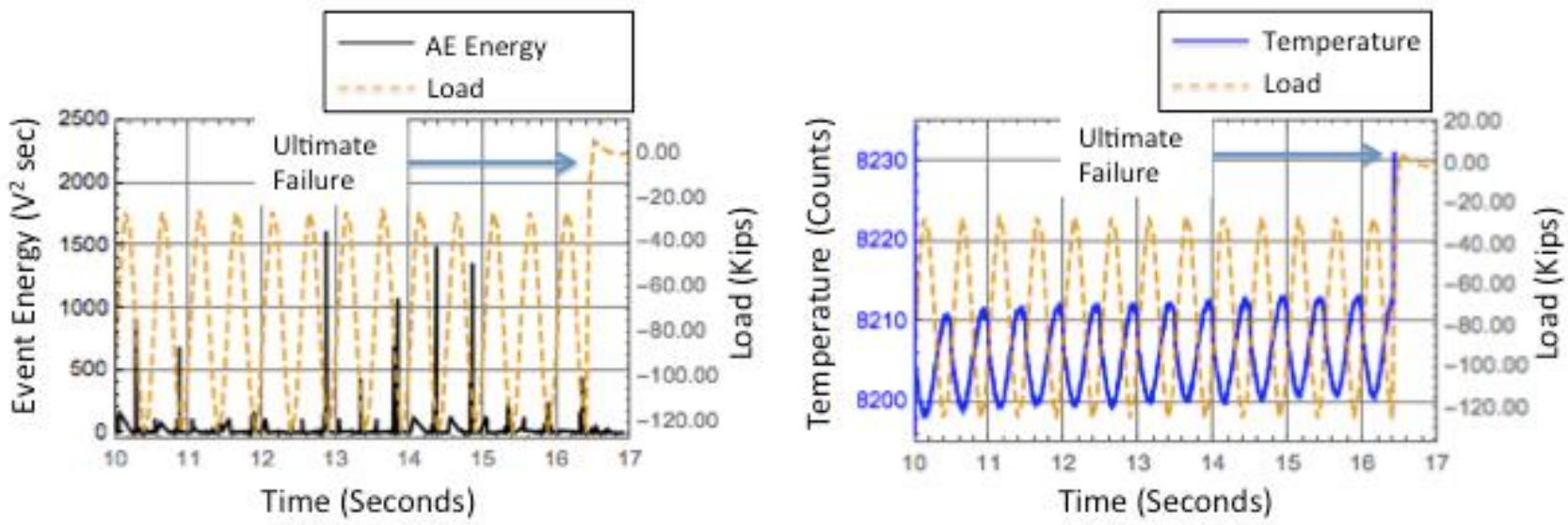

Figure 6. Comparison of AE total energy to applied load (left plot) and comparison of temperature to the applied load (right plot). 
the sensors, are compared to the applied compressional fatigue loading. The acoustic emission events occur during the downward compression as expected. Shown in the right plot of Figure 6 is the comparison of the temperature increase (over an area on the sample with no defect) to the applied load. The temperature rises as the applied load increases. This is due to the thermo-elastic effect where the applied compression causes heating and is 180 degrees out of phase with the loading.

The total event energy is determined by summing the $S E$ values for all the acoustic emission sensors over time. This is shown in left plot of Figure 7. The total event energy is compared to the averaged temperature over delaminated area I1 which is the area where ultimate failure occurred. Shown in the right plot of Figure 7 is the area pixels (estimated damage growth cyclically changing with periodic load) around delaminated area I1 versus the event energy over time. The estimated damage growth is determined by counting the number of "hot" pixels between 22.5 - 30.0 degrees C.

Sudden changes in damage growth related to a previously measured significant acoustic emission event (energy over a $1,000)$ might provide a capability to determine damage depth. The acoustic emission event can provide a time difference between the start of the event to when the thermal energy conducts to the surface and is visible. This time difference can potentially determine defect depth. An example is the circled area in the right plot of Figure 7, where a significant acoustic emission event preceeds a jump in the damage size. Further testing of aditional composite structures is required to study the feasibility of damage depth detection. Both plots of Figure 7, however, show trends of the acoustic energy over time and released thermal energy can be used to identify and track damage development and identify the area of failure within the composite.
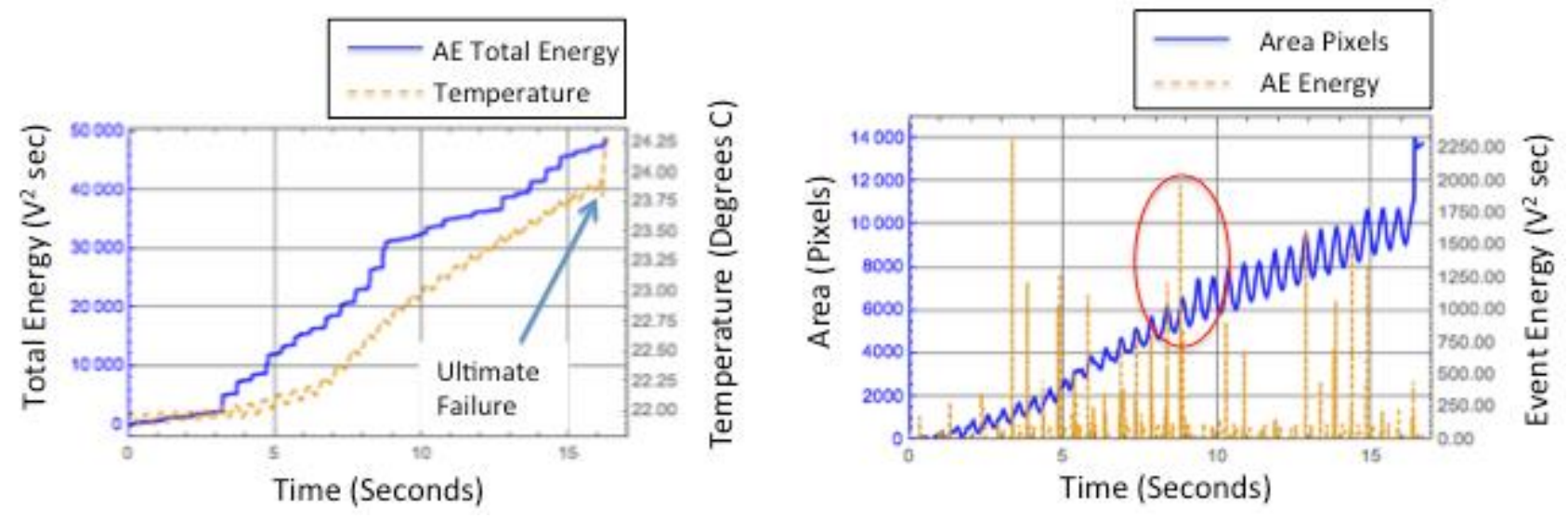

Figure 7. Comparison of total acoustic emission energy to temperature and damage growth to event energy.

\subsection{Processing Thermography Data for Mapping}

An image processing routine was developed to map the acoustic emission data onto the thermal imagery. The procedure is shown in Figure 8 along with some example thermal images. This required improving image contrast, removing angular rotation, and correcting for optical barrel distortion. In addition, the resolution per pixel was determined by measuring know distances on the composite structure. The acoustic emission data were then mapped onto thermal data, revealing the cluster of acoustic emission event locations around the thermal signatures of interest.

The processing technique used to improved defect contrast can be implemented in real time and involves a delayed image subtraction [5,12]. A moving buffer is required in the acquisition software. This delayed subtraction is given as:

$$
\text { Difference Image }=\text { Acquired Image }[i]-\text { Previous Image }[i-k / 2]
$$

where $k=$ number of images per cycle and $i$ is the current acquired image number. Dividing $k$ by 2 ensures the maximum and minimum temperature images are subtracted, within a cycle, and this also provides a series of images with optimal defect contrast varying with image depth. Improved defect signal to noise can be obtained by averaging the 
difference images over a number of cycles if desired. The removal of angular rotation is performed using an affine transformation [13]. The barrel distortion is corrected using an image forward transformation using the equation below [14].

$$
r_{\text {undistorted }}=r_{\text {distorted }}\left(1-d\left(r_{\text {distorted }}\right)^{2}\right)
$$

Where $r_{\text {undistorted }}$ is the corrected Cartesian coordinate distance from the center of the image and $r$ distorted is the distorted

Cartesian distance from the center of the image. The distortion parameter $d$ was set to 0.2 to remove the distortion. The pixel resolution was calculated to be 8.2 pixels per $\mathrm{cm}$. A comparison of the contrast enhanced, rotation corrected and barrel distortion thermal image is shown in Figures $8 \mathrm{~b}$ and 8c. The image in Figure 8c is used to map the acoustic emission data.

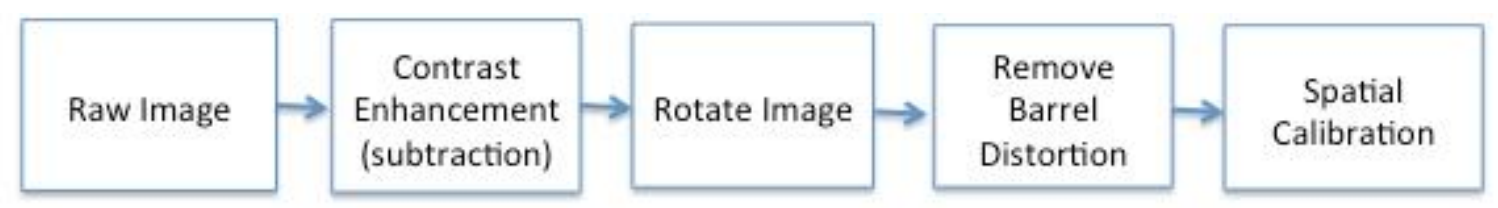

(a) Raw Image

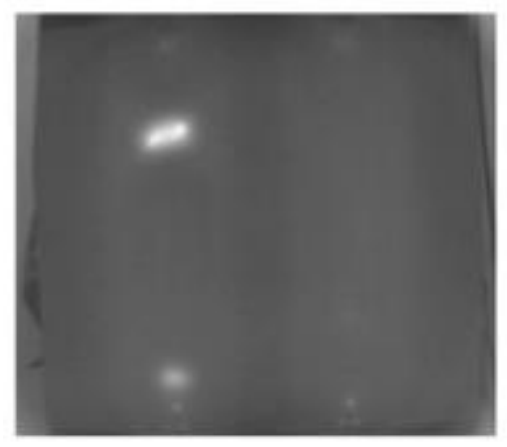

(b) Contrast Enhanced

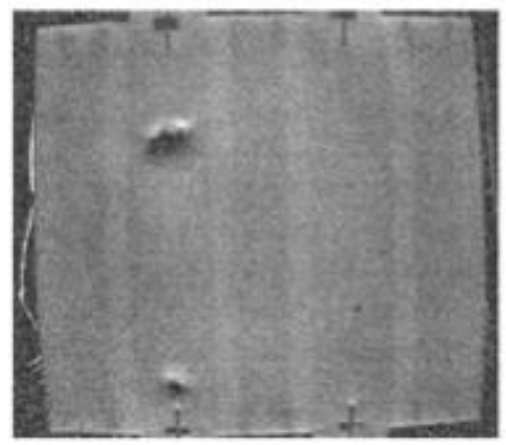

(c) Rotated/Barrel Distortion Removed

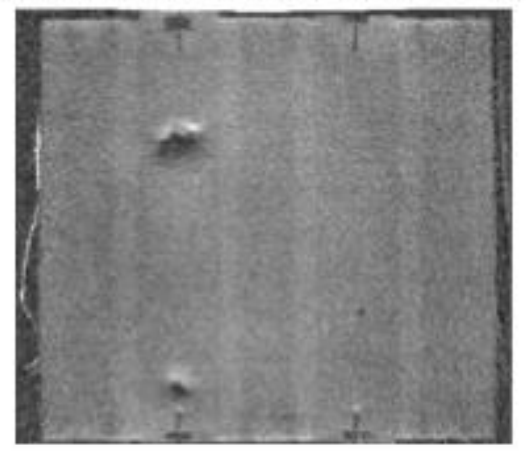

Figure 8. Procedure to process thermal imagery for mapping of acoustic emission data.

\subsection{Acoustic Emission Mapped to Thermography}

The mapped acoustic emission data is shown in Figures 9 and 10 for the flat side and stringer side respectively. The impact locations $(\mathrm{X})$ are also shown. The indicator size and color are based on the energy of the event given by the equation:

$$
\text { Indicator }=\text { Round }\left[(\text { event energy })^{1 / 4}+10\right]
$$

where the event indicator values of less than 13 were given a low energy color of blue (opacity of 0.5 ), indicator values between 13 and 15 were designated as a mid energy event color yellow (opacity of 0.7 ), and greater than 15 was a high energy event with color red (opacity of 1.0). As the structure nears failure, the accumulated acoustic events and indicator values increases as shown in Figure 9. Ultimate failure occurred at the location where the red high energy indicators and mostly yellow indicators clustered. It is important to note the location of the acoustic emission events are prone to errors due to the progression of damage (fiber breaks, delaminations, matrix cracking) as the structure nears failure. These defects can affect the propagation of the acoustic emission signal in terms velocity and waveform mode conversions resulting in processing errors, however, overall on average the clustered location is comparable to the thermal indications of the growing damage areas. In particular for the left image in Figure 10, some of the clustered 

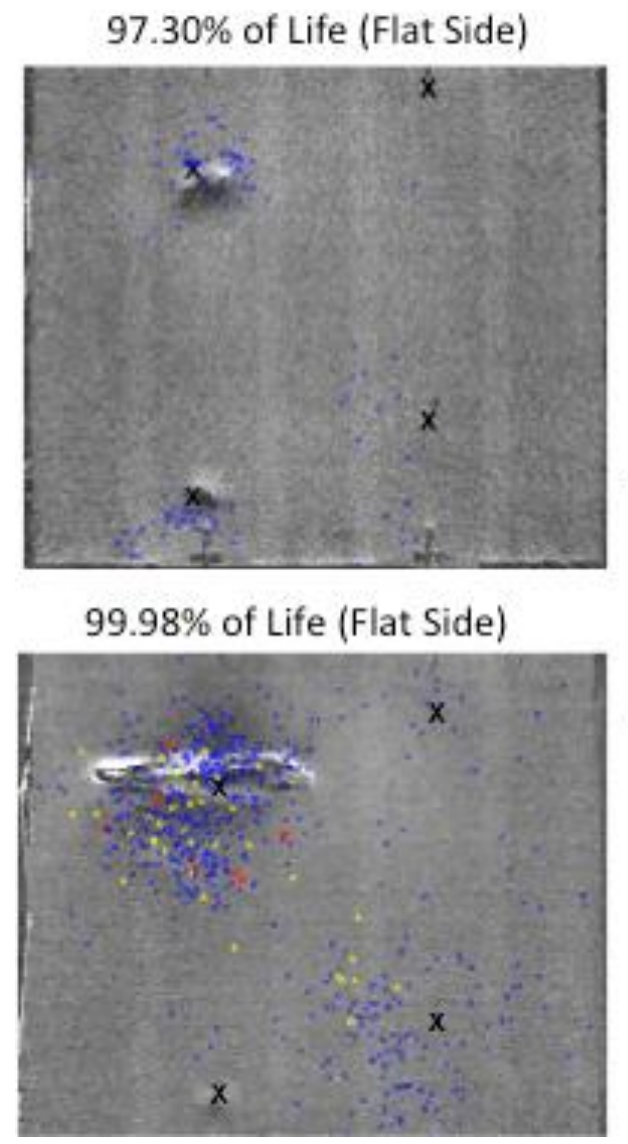

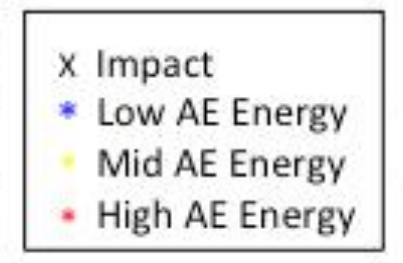

Figure 9. Mapped acoustic emission events onto the process thermal images (flat side).
$99.94 \%$ of Life (Flat Side)
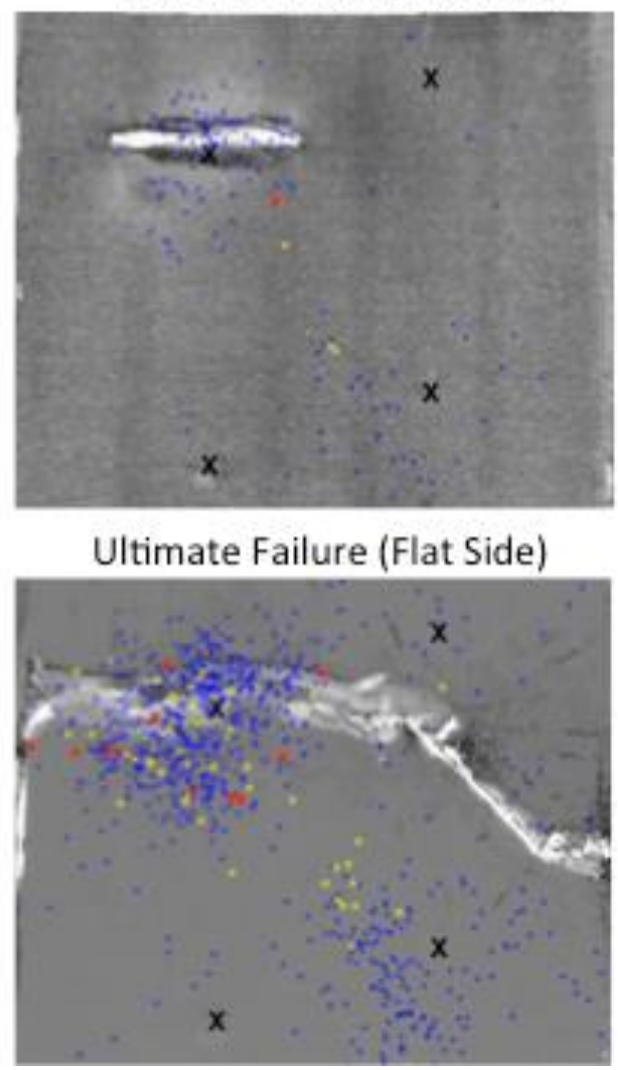

points tends to accumulate below the delamination on the right side. This is due to a growing disbond between the stringer flange and the skin below the delamination.

\section{CONCLUSIONS}

Combining thermography with acoustic emission developed a large area and real time NDE inspection technique for a cyclic fatigue loaded composite. By combining these two inspection techniques, confirmation that a particular area of heating to where acoustic emission events were clustered revealed where damage growth was occurring and where ultimate failure occurred. Synchronizing the acquisition of the thermal and acoustic emission data to the applied load allowed for comparison of heating to the acoustic emission events. Further testing of composite structures is required to study the feasibility of damage depth detection based on significant acoustic emission events and released thermal energy. For this study a static loading test with detected damage would be of interest. Finally, an image processing procedure has been presented to allow mapping of the acoustic emission events onto the thermal images.

\section{ACKNOWLEDGEMENTS}

The authors would like to acknowledge Mr. Wade Jackson of NASA Langley for ultrasonic inspections, sample preparation, and mounting. Also the authors would like to acknowledge Mr. William Johnston of Lockheed Martin for testing support and Mr. Kenneth McLarney, NASA Internship, Fellowship, and Scholarships (NIFS) student for data processing. 


\section{$99.94 \%$ of Life (Stringer Side)}

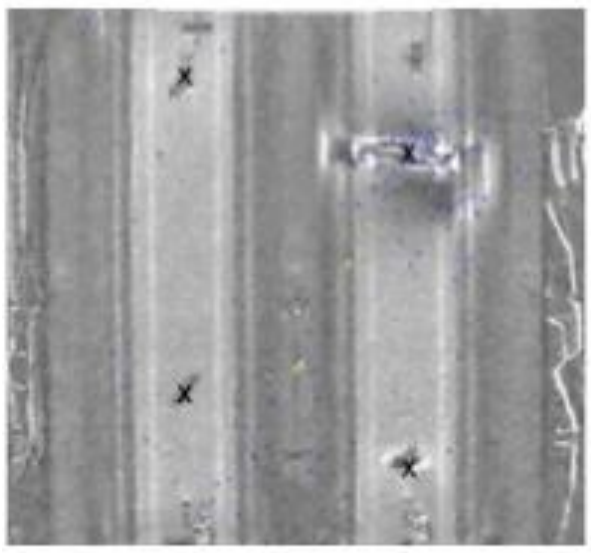

$99.98 \%$ of Life (Stringer Side)

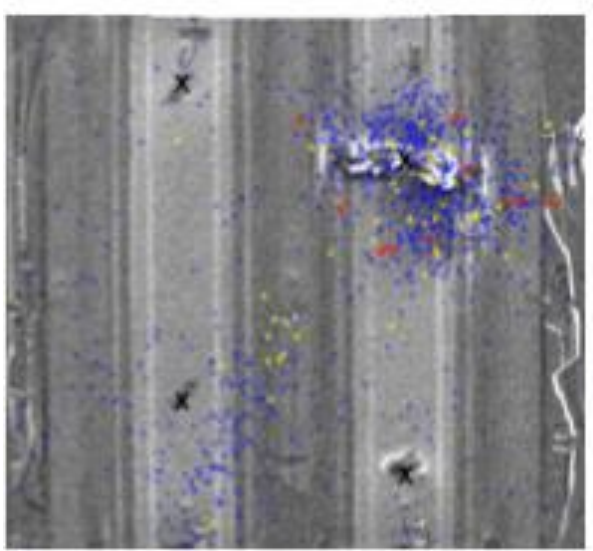

$99.95 \%$ of Life (Stringer Side)

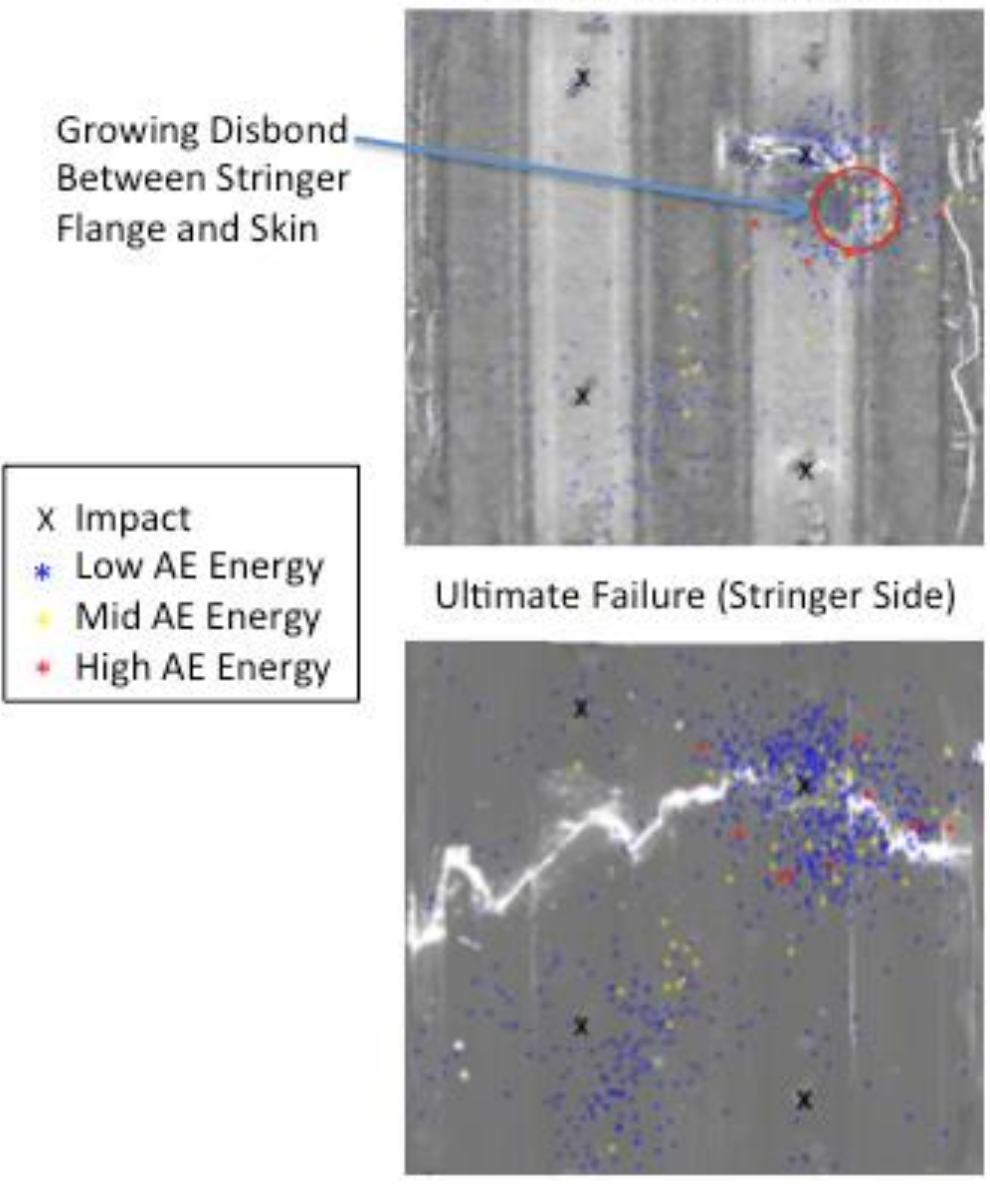

Figure 10. Mapped acoustic emission events onto the process thermal images (stringer side).

\section{REFERENCES}

[1] O'Brien, T. K., "Development of a Delamination Fatigue Methodology for Composite Rotorcraft Structure", NASA Aviation Safety Technical Conference Denver, CO, (October 2008).

[2] Krueger, R., "An Approach to Assess Delamination Propagation Simulation Capabilities in Commercial Finite Element Codes", NASA/TM-2008-215123, 2008.

[3] Bisagni, C., Dávila, C. G., Rose, C., and Zalameda, J. N., "Experimental Evaluation of Damage Progression in Postbuckled Single Stiffener Composite Specimens", American Society for Composites $29^{\text {th }}$ Technical Conference Proceedings, US-Japan 16, ASTM D30, (Feb. 2014).

[4] Zalameda, J. N., Burke, E. R., Parker, R. F., Seebo, J. P., Wright, C. W., and Bly, J. B., "Thermography Inspection for Early Detection of Composite Damage in Structures during Fatigue Loading", Thermosense XXXIV, edited by Douglas Burleigh, Gregory R. Stockton, Proc. of SPIE Vol. 835403 (April 2012).

[5] La Rosa, G., Clienti, T., Savio, F. Lo, "Fatigue Analysis by Acoustic Emission and Thermographic Techniques", Procedia Engineering, XVII International Colloquium on Mechanical Fatgue of Metals, (ICMFM17), Volume 74, pp. 261-268, 2014.

[6] E. Kordatos, K. Dassios, D. Aggelis, T. Matikas, "Rapid evaluation of the fatigue limit in composites using infrared lock-in thermography and acoustic emission”, Mech Res Commun, 54 (2013), pp. 14-20 
[7] Munoz, V., Vales, B., Perrin, M., Pastor, M. L., Welemane, H., Cantarel, A., and Karama, M., "Damage detection in CFRP by coupling acoustic emission and infrared thermography", Composites Part B: Engineering, Volume 85, pp. 68-75, February 2015.

[8] Ringermacher H. I., et al, "System and Method for Locating Failure Events in Samples Under Load", United States Patent No. 7,516,663 B2, (April 14, 2009).

[9] Horne, M. R. "Rayleigh Wave Acoustic Emission during Crack Propagation in Steel". PhD Dissertation, Virginia Polytechnic Institute and State University. (2003).

[10] S. M. Ziola and M. R. Gorman, "Source location in thin plates using cross-correlation", J. of Acoustic Society of America, 90 (5), (1991).

[11] M.G. Baxter, R. Pullin, K. M. Holford and S. L. Evans, "Delta T source location for acoustic emission”, Mechanical System and Signal Processing, 21 (3), (2007).

[12] Winfree, W. P., Zalameda, J. N., and Howell, P. A., "Improved flaw detection and characterization with difference thermography", Proc. SPIE 8013, 80130U (April 2011).

[13] Weisstein, Eric W., "Affine Transformation.”, From MathWorld-A Wolfram Web Resource. http://mathworld.wolfram.com/AffineTransformation.html. (accessed 5 October 2015).

[14] Gribbon, K. T., C. T. Johnston, and Donald G. Bailey. "A real-time FPGA implementation of a barrel distortion correction algorithm with bilinear interpolation." In Image and Vision Computing New Zealand, pp. 408-413. 2003. 